Article

\title{
Technical and Economic Optimal Solutions for Utility-Scale Solar Photovoltaic Parks
}

\author{
Miguel Silva ${ }^{1}$, Rui Castro ${ }^{2, *(\mathbb{D})}$ and Mário Batalha ${ }^{3}$ \\ 1 Instituto Superior Técnico, University of Lisbon, 1049 Lisbon, Portugal; miguel.564@hotmail.com \\ INESC-ID/IST, University of Lisbon, 1000 Lisbon, Portugal \\ 3 Sotécnica, Vinci Energies Portugal, 2664 São Julião do Tojal, Portugal; mbatalha@sotecnica.pt \\ * Correspondence: rcastro@tecnico.ulisboa.pt; Tel.: +351-21-8417287
}

Received: 3 February 2020; Accepted: 26 February 2020; Published: 28 February 2020

check for updates

\begin{abstract}
Solar energy has shown to be a successful renewable source, wherefore large investments are planned in the upcoming decades. This work aims at developing technical and economical solutions to optimize a utility-scale grid connected solar photovoltaic park with an installed capacity of $24 \mathrm{MWp}$. Several successive simulations were automatically performed with the PVSyst software, so that each influencing parameter is individually analyzed. Moreover, a comprehensive study touching relevant aspects, such as central versus string inverters, landscape versus portrait dispositions, the optimal tilt angle (for fixed tilt systems), backtracking strategy (for single axis tracking systems), shading limit angle, and pitch is performed with the objective of finding the configuration leading to the higher grid injected energy output. The levelized cost of energy (LCOE) was obtained for 12 different cases in which, for both fixed tilt and single-axis tracking (SAT), central and string inverters and portrait/landscape disposition were considered. The lowest LCOE achieved was 32.23€/MWh, which is a clear indication of the competitiveness of the solar Photovoltaic (PV) technology. The most economic viable solution is characterized by the employment of an SAT system with the backtracking strategy, together with string inverters and module portrait disposition.
\end{abstract}

Keywords: utility-scale solar PV park; optimization; design parameters; string inverters; economic analysis; levelized cost of energy (LCOE)

\section{Introduction}

Energy is crucial for a wide range of applications in agriculture, transportation, industry, and household sectors, among others. The continuous consumption of fossil fuels for power generation is leading to large amounts of greenhouse gas emissions, which heavily harms the environment. Among many consequences, the global warming and climate change have been identified as an unsettling phenomenon, which requires mankind to reverse this process as soon as possible. In this regard, the employment of renewable energies appears to be one of the foremost efficient solutions.

In the past few years, solar energy has shown to be a promising solution, since the installation is accessible, and it can be extended to any place where there exists abundant sun radiation. The strong reduction of the investment costs along with the diverse components' efficiency improvements will make the installed power to keep growing in the future, namely through the implementation of large-scale grid connected solar PV parks. Hence, the optimization of solar PV parks turned out to be a crucial topic to be studied, since it highly influences the energy output and the project's economic viability in the long term. The solar PV park optimization is a not a straightforward problem due to the existence of many variables that need to be considered, which might result in parameters with controversial values. 
Throughout time, several studies concerning the performance assessment of solar PV parks have been carried out aiming at the attainment of greater performance indexes. Furthermore, research in the field of solar PV power plants optimization has been undertaken, as this represents a multivariable problem involving several parameters that must be considered. The optimization domain is a crucial matter that has been gaining particular importance in the past few years, since PV technology costs have been noticeably decreasing and large-scale parks have been and are being built. In this context, just small improvements may lead to important yield performance differences.

In [1], a grid-connected solar PV power plant performance was assessed through PVSyst software. Performance ratio and various types of power losses data were obtained and from it, the viability of installing a 1 MWp solar PV power plant in four different areas was discussed. The solar modules' tilt angle was adjusted accordingly to the site latitude, and both the modules and inverter assumed throughout the study were initially chosen, having been the only option considered.

A design simulation was carried out in paper [2] aiming at the performance assessment yield and loss forecasting for a $100 \mathrm{kWp}$ grid-connected solar PV system. Throughout the study, an equivalent mathematical model was developed, and the performance parameters were obtained with the help of the PVSyst design tool. Similarly, the study developed in [3] aimed at the design and simulation of a $1 \mathrm{kWp}$ solar PV system power plant using PVSyst software. The energy generated by the system and incurred losses were computed and analyzed. As it does not represent a large-scale system, the modules' tilt angle was simulated as being varied seasonally accordingly to the "optimal angle" provided by the software. As in [1], both the module and inverter type were initially chosen. Paper [4] presents a similar study to [3], evaluating the performance of a $10 \mathrm{MWp}$ PV power plant to be installed in a given area of 50 acres while also considering seasonal tilt angle variation.

In [5], a study of a 150 MWp grid-connected utility-scale solar PV plant to be installed within a 750 acres land is presented. The analysis was mainly focused on the preliminary design of the case project such as the feasibility study and PV solar design aspects, which were underpinned by an energy yield assessment simulation study performed using PVSyst software. The fixed tilt angle was also set accordingly to the site coordinates. In [6], a research study was carried out, in which several performance parameters, such as Performance Ratio (PR), utilization factor, and factors contributing to the performance of solar power plants (climate conditions, design parameters) are covered, which is essential in the extent that it helps when trying to optimize the energy generation and the PV park overall performance. We recall that PR is the ratio of the energy effectively injected in the grid, with respect to the energy that would be produced if the system was continuously working at its nominal STC efficiency. STC stand for Standard Test Conditions, i.e. laboratory conditions under which the solar PV panels are tested to determine the module nominal efficiency, among other parameters. The following are the requirements for standard test conditions: irradiance $=1000 \mathrm{~W} / \mathrm{m}^{2}$; module temperature $=25^{\circ} \mathrm{C}$; air mass 1.5 (AM 1.5) spectrum.

In [7], an analysis of the mutual shading effect in rows arrangement is undertaken using PVSyst software, which plays a major role in a fixed tilt solar PV park optimization. A deep analysis of the different components' shading effects (beam, diffuse, albedo, and mismatch electrical effects) was carried out, and it was concluded that the diffuse and albedo losses are dominating. Furthermore, in [8], the same author introduced a tool to optimize the layout of ground-based PV solar systems considering economic boundary conditions. Besides the optimization aiming at maximizing the installation yield performance, economic boundary conditions such as the investment and maintenance costs, surface availability, and feed-in tariff have also a relevant impact on the design choices. So, the optimization tool addresses the mentioned problem by finding the ground cover ratio and module tilt angle that is capable of optimizing the economic benefit.

A cuckoo search-based algorithm was developed [9] aiming at the sizing optimization of a $5 \mathrm{MW}$ large-scale grid connected PV system. Over this study, the algorithm was used to select the optimal combination of the system components (PV module and inverter) so that the PR was maximized. In [10], a genetic algorithm was developed for the calculation of the optimal configuration of large 
PV plants aiming at minimizing the levelized cost of energy (LCOE). The design optimization was carried out considering the number of components and their arrangement within the field as well as the lifetime cost and energy production. The design results allowed concluding that the proposed optimization method leads to a reduction of the energy cost generated by the PV plant, enabling the economic benefit maximization.

Paper [11] considers the effect of azimuth and tilt of fixed solar PV systems considering total energy, economics, and peak power implications. The results challenge the default placement ruleof thumb. The impact of tilt and azimuth on PV energy yield is analyzed for Saudi Arabia in [12]. The authors claim that adjusting the orientation 5 times/year increases the energy yield by $3.63 \%$. The impact of a single-axis tracking (SAT) PV system on energy scenarios has been studied, as seen in [13], where it is concluded that the inclusion of SAT decreases global average LCOE by $6 \%$. In paper [14], a procedure is presented for optimizing the electrical configuration of a PV array. The performance of the optimization system is evaluated in partial shading conditions using a SPICE (Simulation Program with Integrated Circuit Emphasis) circuit simulator. Methods to optimize inverter configurations for grid-connected PV systems have been proposed, as it is the case of [15], where the inverter configuration and operation strategy impacts on the LCOE are assessed.

In a more general view, the continuous implementation of more and more solar PV power installations in the power grids is driving the need for optimization approaches in the smart-grid context. In [16], a multi-objective evolutionary algorithm for demand-side management with load-balancing approach is proposed. The annual power load forecasting is a delicate problem which is approached in [17] by using a hybrid method combining a generalized regression neural network and fruit fly optimization algorithm.

As solar PV power is spreading all over the power grids, Distribution System Operators (DSOs) are becoming more and more interested in solar PV power forecasts as a tool to increase the reliability and security of their grids. In recent years, statistical and machine learning-based energy prediction methods have been given great attention in the literature. In [18], a spatial-temporal forecasting method based on the vector autoregression framework, which combines observations of solar generation collected by smart meters and distribution transformer controllers, is presented. The general subject of time-series prediction is approached in [19] by proposing a distributed algorithm that performs density-based clustering and exploits the identified clusters to solve both single- and multi-target regression tasks. The same authors apply a method [20] that learns artificial neural networks to PV power forecasts. The method performs online adaptive training and enriches the entropy measures with spatial information of the data. Deep learning techniques are also being applied to forecast PV power as it is the case of [21]. In this paper, the use of a long short-term memory recurrent neural network (LSTM-RNN) to forecast the output power of PV systems is shown to be effective because of their recurrent architecture and memory units.

As seen, there are several studies concerning the performance assessment of solar PV parks, in which specific sites are analyzed with a predetermined system. Therefore, these studies are carried out through simulations aiming at comparing all the different sites under analysis, and they do not provide an optimization regarding important parameters to consider, since these are initially set. On the other hand, research can be found specifically covering the optimization problem, as it is the case of some articles included in the state-of-the-art part of our paper. The mentioned references that used the PVSyst as a simulation software tool are indeed relevant studies from an optimization standpoint as well, but these do not consider a methodology to assess the influence of several parameters that the present research encompasses. This research provides a structured generic method aiming at the solar PV park energy yield maximization through successive simulations that allow varying the parameters of interest. Thus, it provides a holistic approach to the initial problem, as it considers several parameters that highly influence the final output at stake. Furthermore, besides the energy maximization, the conducted research also includes an economic analysis for each different prior analyzed situation where several combinations are considered, which allowed us to draw conclusions 
about the most economic viable solution. The latter topic is relevant as nowadays it shows to be inherent to any project, proving that the obtained system leading to the highest energy yield is not the one leading to the lowest levelized cost of energy, being this conclusion highly dependent on the considered detailed data.

Differently from what was described in the mentioned articles, this paper approaches the multivariable optimization problem through a generic method to optimize the parameters deemed relevant in both fixed tilt and single-axis tracking (SAT) PV utility-scale installations. Simulations are carried out aiming at finding the module/inverter combination leading to the higher energy output so that the pair with the best performance could be selected. Different central and string inverters are included in the study so that their performance could be assessed and compared in both technical and economic terms. The inclusion of string inverters in our study is to be highlighted, since they have been recently challenging the traditional central inverters.

Moreover, a comprehensive study touching relevant aspects, such as the landscape versus portrait dispositions, the optimal tilt angle (for fixed tilt systems), backtracking strategy (for SAT systems), shading limit angle, and pitch is performed with the objective of finding the configuration leading to the higher grid injected energy output.

In our paper, we develop optimal solutions for solar PV parks by analyzing the influencing design parameters. We do not use the classical optimization problem formulation with restrictions; instead, we run PVSyst software successively for changing the values of the relevant design parameters and extract conclusions from this analysis. In this regard, we developed a dedicated automatic procedure to run PVSyst successively for different input parameters. From the best of the authors knowledge, this is an innovative added value, as it allows assessing the sensitivity of the output quantities for virtually every value of the input parameters. Through this systematic study, we are able to specify the optimal design parameters of the solar PV park. The goal of the paper is to offer a methodology to optimally design a solar PV park by inspecting all the possible options.

This work aims at developing technical and economical solutions to optimize a utility-scale grid-connected solar photovoltaic park with an installed capacity of $24 \mathrm{MWp}$ and a limited land area of approximately 50 hectares. To that end, the maximization of the energy to inject into the grid will be attained through seeking the most appropriate parameters' values, which will be varied through successive simulations automatically performed by the PVSyst software. Furthermore, along with the energy yield maximization, an economical study is carried out, so that the most viable solution can be chosen. It is noted that the system costs are "real world" values, since they have been provided by the PV market suppliers.

The paper is organized as follows. In Section 2, the used strategy to conclude about the selection of the PV module and different inverters to employ is presented as well as the approach to optimize the fixed tilt and single-axis tracking systems. The economic model is also described, as it was the measure of comparison between the different studied alternatives. In Section 3, the results and discussion section, the main study findings stemming from the performed simulations are depicted. Finally, the conclusions provide a resume of what was found along the study as well as the recommendations of what equipment and configurations should be employed to maximize the energy yield.

\section{Methodology}

Initially, the strategy path taken to conclude about the modules and inverters to employ will be shown. Afterwards, both fixed tilt and SAT systems will be considered in the extent that key parameters will be introduced, as they highly influence the variable to maximize. Finally, the method also introduces the LCOE model, which proves to be extremely useful to compare the different cases.

It is worth mentioning that irradiance and temperature data for performing the simulations were gathered from the PVSyst database for the specific installation site. 


\subsection{Equipment's Selection Strategy}

In an early stage of this study, it was prominent to decide the main devices to employ so that the simulations could be performed with a particular type of configuration, which subsequently allowed other parameters of interest to be tested.

There exist different PV inverters classified in different groups [22], which influence the type of possible connections to be made between the PV modules themselves. In this research, two types of inverters have been investigated:

- String inverters-operate typically between a 0.4 and $2 \mathrm{~kW}$ range for a single string of PV modules, suiting small rooftop PV power plants. This type of inverters started to be recently used in utility-scale PV parks as well, with capacities in the range of hundreds of $\mathrm{kW}$.

- Central inverters-operate in the $100 \mathrm{~kW}$ to $6 \mathrm{MW}$ range with three-phase topology and modular design for larger power plants.

Two different string inverters manufactured by Huawei will be included in this study to conclude about its capability of increasing the energy yield when compared to a central inverter. One clear advantage of employing this type of inverter is the redundancy factor, since if one inverter gets damaged, only a few strings will be affected rather than one entire sub-array. Additionally, replacing a string inverter is much simpler, as it does not require specialist personnel and spare inverters can be kept on site prepared to be used. On the other hand, the employment of this type of configuration will lead to a large number of inverters, as utility-scale systems might be composed by thousands of module strings.

\subsubsection{Central Inverter}

Initially, the number of sub-arrays (number of arrays in which the total PV park is divided) must be chosen, which subsequently influences the type of module and central inverter to select. For the power conversion to be performed at maximum efficiency, it is crucial to know both the DC peak power produced by the PV sub-array as well as the maximum AC power that the inverter is able to deliver. The DC to AC ratio, or oversizing ratio, is known as the ratio of the sub-array DC peak power (at STC) to the inverter's nominal AC power output.

The central inverter to be used hinges on the total power of each sub-array and on the deemed DC to $A C$ ratio interval. The central inverter minimum, $P_{\min , i n v, A C}$, and maximum, $P_{\max , i n v, A C}$, admissible AC power for each different case (where a different number of sub-arrays is considered) might be found based on the inverter maximum, $P_{\text {inv, max }}$,ratio, and minimum, $P_{\text {inv, } m i n, \text { ratio, }}$ DC to AC ratio, which is respectively given by:

$$
\begin{aligned}
& P_{\text {min }, \text { inv }, A C}=\frac{P_{\text {subarray }, S T C, D C}}{P_{\text {inv }, \text { max }, \text { ratio }}} \\
& P_{\text {max }, \text { inv }, A C}=\frac{P_{\text {subarray }, S T C, D C}}{P_{\text {inv }, \text { min }, \text { ratio }}}
\end{aligned}
$$

where the $P_{\text {subarray, } S T C, D C}$ (sub-array DC peak power, i.e., at Standard Test Conditions) varies depending on the number of sub-arrays. An interval between $P_{\text {inv, min, ratio }}$ and $P_{\text {inv, max, }}$,atio is required for the inverter power intervals to be found, which in this study was decided to be within 1.1 and 1.3, respectively, based on [23]. Once the number of sub-arrays to test is known and the $P_{\text {inv, ratio }}$ interval range is decided, the initial available central inverters can be selected.

Once the PV modules and available central inverters are selected, the goal is to carry out a great number of simulations in which all these two types of devices, available in the PVSyst components database, are combined one with each other, aiming at drawing conclusions regarding the performance of each combination. Thereby, for each case where a different number of sub-arrays is considered, all modules will be matched with all available inverters (selected based on the DC to AC ratio). 


\subsubsection{String Inverter}

As the power of a string inverter is much lower in comparison to a central inverter, the solar park does not need to be split into several high-power sub-arrays. In contrast, each string inverter is connected to a few strings, which will result in a larger number of inverters. Again, simulations are to be performed for every combination of module and string inverter. Two recent string inverters manufactured by Huawei were tested, and the respective data are introduced in PVSyst.

\subsection{Fixed Tilt Definitions}

The sheds arrangement is mainly characterized by the collector width $(W)$, pitch (Pitch), and tilt angle $(\beta)$, being this nomenclature presented in Figure 1.

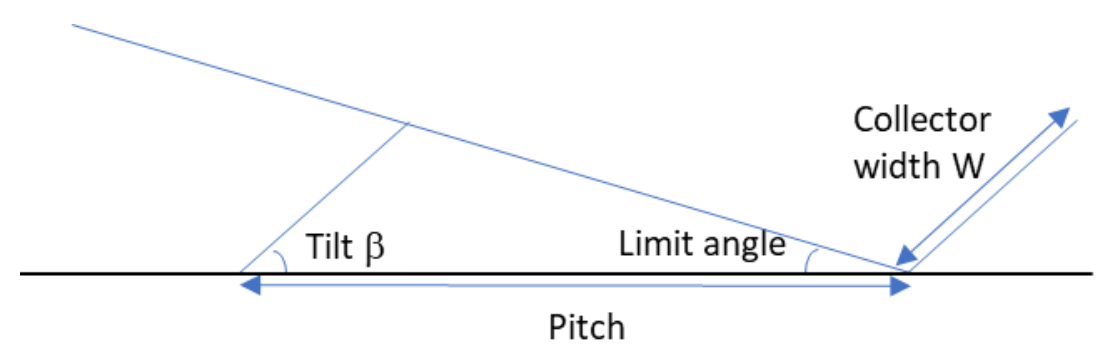

Figure 1. Sheds' scheme.

The shading limit angle $(\theta)$ is the angle from which a shadow starts to be produced on the next shed (see Figure 1). There is no optimum value for this angle, but often one considers the worst-case scenario (December 22) for mutual shadings to be minimized. For a given shading limit angle, the pitch is computed through Equation (3) so that no shadings are produced on the solar panel plane [7].

$$
\text { Pitch }=W\left(\cos \beta+\frac{\sin \beta}{\tan \theta}\right)
$$

The ground cover ratio (GCR) is one of the foremost parameters to regard while designing a PV park, being given by the ratio of the modules' sensitive area to the ground total area occupied by the PV park. However, the "real" ground cover ratio may also be presented through the ratio of the width to the pitch, being the one used in the design of PV parks, as it is based on the shed parameters and not on the initial available area.

\subsubsection{Portrait versus Landscape}

The solar panels' disposition was studied taking into account two different possible solar panels' arrangement: portrait and landscape. It is important to highlight that shading is a factor to consider when optimizing a PV array, as the beam component has an influence on the electrical shading part of the system. Hence, several simulations were performed varying the plane tilt angle for these two different arrangements, therefore allowing to conclude about the shading impact on each case individually.

\subsubsection{Plane Tilt, Pitch, and Azimuth}

When employing a fixed tilt system, the annual energy yield and PR strongly depend on both pitch and plane tilt, as they influence both incident irradiation and shading in the collector plane. We recall that PR is the ratio between the effectively produced energy and the energy that would be produced if the solar panel works at its nominal efficiency, i.e., at STC. An initial sensitivity analysis was carried out to perceive the influence of the tilt angle and pitch on the grid-injected energy and $\mathrm{PR}$, which allowed concluding that the tilt angle that leads to the maximum energy yield is often not equal to the site latitude (as assumed in several studies) but lower than this value due to the mutual shading effect. 
Figure 2 shows an example of the change of the energy yield and PR as a function of the plane tilt. The "Energy (STC)" curve depicts the energy that would be produced if the solar panel operates always at its nominal efficiency (STC). It is apparent that the angle that would lead to a maximum theoretical energy yield is $34^{\circ}$ (without considering shading effects), whereas a plane tilt of $30^{\circ}$ would lead to the effectively maximum energy injected in the grid. Regarding the PR (right scale), one can see that the maximum value is given for the minimum tilt angle, which supports that the optimal system hinges on what is to be maximized. For this specific case, when there is an increase of the tilt angle, the global incident irradiation will increase significantly. However, the mutual shadings will also increase, which in turn will lead to a decrease of the grid energy and, consequently, a degrading of the performance ratio.

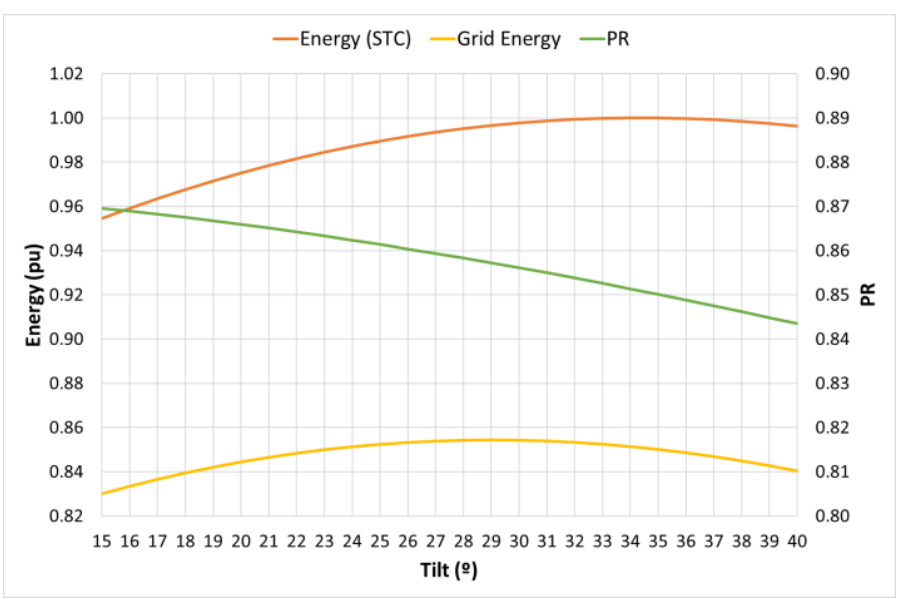

Figure 2. Example of Energy (Standard Test Conditions, or STC), grid-injected energy and Performance Ratio (PR) as a function of tilt.

Relative to the azimuth angle, in the northern hemisphere, it is common for the plane to face south $\left(0^{\circ}\right.$ azimuth angle) to receive the maximum amount of irradiation.

\subsection{Single-Axis Tracking Definitions}

When using single-axis trackers, near shadings have always to be considered when employing or not the backtracking strategy. In the former case (with backtracking), instead of tracking the sun so that the solar modules are always perpendicular to the beam irradiance (without backtracking), these will adopt a lower angle (in the morning and evening) to avoid beam mutual shadings, which also makes the incidence angle increase. Thus, when making use of this strategy, the shading electrical loss effects are reduced, but the misorientation losses increase. The factor that might lead one to adopt the backtracking strategy is the lower electrical shading effects, since when a part of a string gets shaded, the full production from that string is affected. Simulations considering both strategies were performed under the same conditions to draw results regarding the best one to employ.

As in the fixed tilt configuration, the distance between trackers (pitch) highly influences the energy yield. After a certain pitch, the energy yield does not vary significantly, and the ground cover ratio may be obtained as in the fixed tilt configuration.

\subsection{Economic Viability}

Besides the technical optimization through the equipment choice and parameters' variation, the economic viability analysis of each different studied case is to be carried out as well. To that end, 
the levelized cost of energy (LCOE) may be computed for every single case. The LCOE ( $€ / \mathrm{MWh}$ ) is the discounted average cost per unit of electricity produced during the solar PV park lifetime:

$$
L C O E=\frac{T C}{E_{a}}=\frac{F C+V C}{E_{a}}
$$

where $T C$ is the total annual cost $(€)$ given by the sum of the discounted annual fixed cost $(F C)$ and annual variable cost $(V C)$. The variable cost is the cost parcel that changes with the production size. It includes the fuel cost, the cost of $\mathrm{CO}_{2}$ emissions, and the Operation \& Maintenance (O\&M) costs that depend on the production size. In PV systems, there is no fuel cost, nor $\mathrm{CO}_{2}$ emissions; furthermore, we have considered that $O \& M$ costs may be accounted for as a percentage of the investment, so we assumed they do not depend on the production size. Therefore, the variable cost is considered to be zero. The fixed cost $F C$ include both the initial investment $I_{0}$ and the O\&M costs over the power plant's lifetime. The former one can be written as a function of a constant annual payment (annuity $-A_{T}$ ) over the project's lifetime:

$$
I_{0}=A_{T} \sum_{j=1}^{n} \frac{1}{(1+r)^{j}}=A_{T} \frac{(1+r)^{n}-1}{r(1+r)^{n}}=\frac{A_{T}}{\alpha} .
$$

In Equation (5), $r$ and $n$ are the minimum rate of return and the project's lifetime, respectively. Summing both the annuity payment and the fixed O\&M costs (which is assumed to be proportional to the investment by a factor $\beta$ ), one can obtain the fixed costs as:

$$
F C=(\alpha+\beta) I_{0}
$$

\subsection{Case Studies Summary}

Twelve different cases were regarded throughout the study, enclosing significant PV system's variations. More specifically, each combination considers a different type of inverter (string versus central), solar panel configuration (portrait versus landscape), and type of tracking system (fixed tilt versus single-axis tracking). Therefore, the energy yield maximization as well as the subsequent LCOE assessment were performed for these 12 distinct cases that account for different matching possibilities.

\section{Results and Discussion}

\subsection{PV Module and Inverter Selection}

\subsubsection{Central Inverter}

We recall that in the considered application the total installed DC capacity is $24 \mathrm{MWp}$, and the $\mathrm{DC}$ to AC ratio may change between 1.1 and 1.3. This allowed defining the inverters' maximum and minimum capacity and eventually selecting the central inverters that could possibly suit each considered number of sub-arrays. We decided to restrict our analysis to 7 and 8 sub-arrays, as for less sub-arrays, one big damaged inverter, combined with the fact that the replacement needs specialized personnel, would lead to large yield losses. After the selection of the proper central inverters from the PVSyst database and picking up all the available PV modules in the same database, a high number of simulations were carried out under the same conditions to match all possible module/inverter combinations. The top three best solutions for each number of sub-arrays are presented in Table 1 , this selection being based on the maximum annual grid injected energy $\left(E_{\text {grid }}\right)$. 
Table 1. Top module/central inverter combinations leading to the higher grid injected energy; seven and eight sub-arrays.

\begin{tabular}{cccc}
\hline No Sub-Arrays & Module & Inverter & E $_{\text {grid }}$ (MWh) \\
\hline \multirow{2}{*}{7} & Hanwha Qcells 365 W & SunGrow SG3000HV & 37,537 \\
& Hanwha Qcells 370 W & SMA Central 2750 & 37,798 \\
& Hanwha Qcells 370 W & SunGrow SG3000HV & $\mathbf{3 8 , 0 9 3}$ \\
\hline \multirow{2}{*}{8} & Hanwha Qcells 370 W & SMA Central 2750 & 37,841 \\
& Hanwha Qcells 370 W & SMA Central 2500 & 37,920 \\
& Hanwha Qcells 370 W & SunGrow SG2500HV & $\mathbf{3 8 , 0 8 6}$ \\
\hline
\end{tabular}

As one may conclude, the $370 \mathrm{~W}$ module (Hanwha Qcells) combined with the central inverters SG3000HV and SG2500HV manufactured by SunGrow make up the best-case scenarios for 7 and 8 sub-arrays, respectively. Following the reasoning related to the highest possible number of sub-arrays (less losses in case of a failure), the combination leading to a greater grid energy yield for 8 sub-arrays (Hanwha Qcells $370 \mathrm{~W}+$ SunGrow SG2500HV) was found to be the best option, the energy output being marginally smaller than for the 7 sub-arrays case.

\subsubsection{String Inverter}

Although there is no need to divide the park according to the number of inverters (sub-array), one amongst the 8 aforementioned sub-arrays was used to perform the simulations with the string inverters. The simulations were undertaken considering a maximum DC power of $3000 \mathrm{kWp}$ and the same DC to AC ratio interval (1.1 to 1.3). In addition, a sub-array configuration of 29 modules in series and 280 strings in parallel was considered. This was the configuration of the sub-array that lead to the maximum energy yield, according to the simulations performed. The string inverters data that allowed to use PVSyst to simulate the string inverters were kindly supplied by the manufacturer. Table 2 depicts the top sub-array grid injected energy $\left(\mathrm{E}_{\text {grid }}^{\prime}\right)$ results as a function of the number of inverters per sub-array. The two string inverters under analysis are the Huawei SUN2000-105KTL $(105 \mathrm{~kW})$ and SUN2000-185KTL (185 kW).

Table 2. Top module/string inverter combinations leading to the higher sub-array grid injected energy; different number of inverters per sub-array.

\begin{tabular}{cccc}
\hline $\mathbf{N}^{\circ}$ Inv. & Module & Inverter & $\mathbf{E}_{\text {grid }}^{\prime}(\mathbf{M W h})$ \\
\hline 26 & & & 5010 \\
25 & & & 5010 \\
24 & Hanwha Qcells 370 W & SUN2000-105KTL & 5010 \\
23 & & & 5010 \\
22 & & & 5008 \\
\hline 15 & \multirow{2}{*}{ Hanwha Qcells 370 W } & SUN2000-185KTL & 5002 \\
14 & & & 5002 \\
\hline
\end{tabular}

Again, in the case of the string inverters, the module Hanwha Qcells $370 \mathrm{~W}$ leads to the best results, but Table 2 shows that the energy yield is very close in all cases. So, decisions are to be taken based on costs. The energy yield difference between using 22 or 26 SUN2000-105KTL inverters per sub-array is only $0.04 \%$, which is clearly not enough to offset the initial investment of four string additional inverters per sub-array. So, the economic assessment will consider 22 inverters per sub-array of this type, making a grand total of 176 string inverters of $105 \mathrm{~kW}$ each. For the same reason, and since the energy yield difference between using 14 or 15 SUN2000-185KTL string inverters makes up only $0.012 \%, 14$ string inverters per sub-array (totaling 112 string inverters of $185 \mathrm{~kW}$ ) will be considered in the economic analysis. 
We note that the differences in the results from Tables 1 and 2 stem from the applied simulation methodology. Tables 1 and 2 present the best results (i.e., highest energy yield) obtained through successive simulations for different solar module and inverter combinations. Thus, the undertaken successive simulations matched a different solar module/inverter combination making use of each device's datasheet (provided both by PVSyst and by the string inverter manufacturer, as this is a novel technology).

\subsection{Fixed Tilt Energy Maximization}

We have compared the performance of the portrait and landscape configurations regarding the sub-array grid injected energy. The portrait and landscape dispositions were considered to have two modules vertically oriented and four modules horizontally oriented along the shed's width, respectively. Figure 3 depicts the sub-array grid injected energy as a function of the tilt angle (with a constant pitch equal to $9.65 \mathrm{~m}$ ), for both the landscape and portrait dispositions, using the central inverter configuration. When the tilt angle increases, more shadings will be produced in the collector plane, causing a decrease in the produced energy as well. Landscape disposition produces more energy, so it will be employed henceforth to analyze the other different parameters while seeking the maximization of the energy yield.

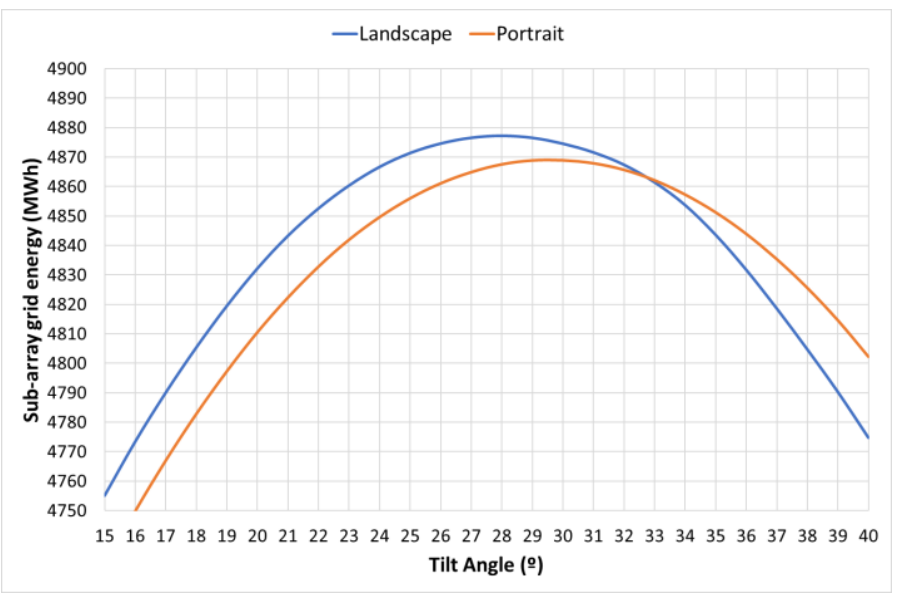

Figure 3. Sub-array grid injected energy as a function of tilt; portrait versus landscape; central inverter; pitch $=9.65 \mathrm{~m}$.

We note that different string cables' length are required depending on whether the panels are disposed in portrait or landscape. Thus, no conclusions can be directly drawn from this analysis regarding the most economical viable configuration to employ, as different dispositions lead to different energy outputs but also to different initial investments.

Through the pitch and tilt angle, one must ensure that the shadings stemming from adjacent rows are minimized as much as possible. A shading limit angle of $18^{\circ}$ was set for no shadings to be produced between 9 and $16 \mathrm{~h}$ on December 22 (worst case). Figure 4 depicts the sub-array grid injected energy and the pitch (right scale) as a function of the tilt angle for the landscape disposition and central inverter configuration.

If the shading limit angle is to be kept constant, the pitch and tilt angle are dependent on each other. So, a tilt angle increase will be followed by a pitch increase (as can be seen in Figure 4) for the shading limit angle to remain constant. As pictured in Figure 4, there exists a maximum of produced energy corresponding to a specific pitch and tilt angle. Below and above $30^{\circ}$, the beam and diffuse irradiance hitting the solar modules decrease, leading to the shape of the blue curve. The exact values leading to the maximum energy yield are given by a tilt angle of $30^{\circ}$ and a pitch of $9.65 \mathrm{~m}$, which results in a GCR of $41.45 \%$. 


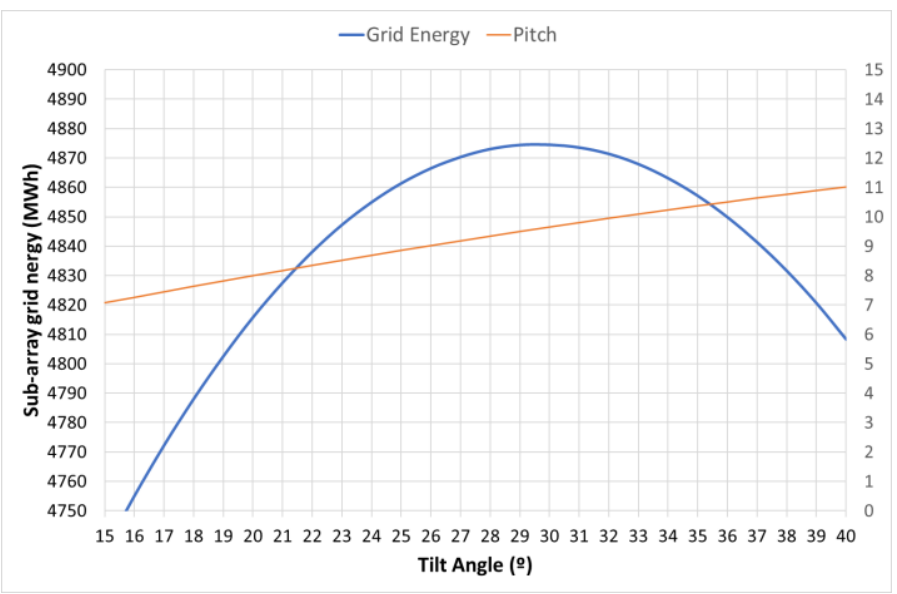

Figure 4. Sub-array grid injected energy and pitch variation as a function of tilt; $18^{\circ}$ shading limit angle; landscape; central inverter.

Simulations for all possible combinations of pitch and tilt angle without limit angle restrictions were carried out to conclude about their influence on the grid injected energy. Figure 5 depicts the sub-array grid-injected energy results concerning the variation of both parameters, pitch and tilt, for the landscape disposition and central inverter configuration. As it can be seen, both pitch and tilt angle start to increase initially for the energy yield to be maximized. The tilt angle tends to become steady around $30^{\circ}$ for a pitch of approximately $10 \mathrm{~m}$, after which the grid energy reaches the maximum and does not vary significantly for higher pitch values.

In Figure 6, the sub-array grid energy output along the pitch are presented for a fixed tilt angle of $30^{\circ}$ (corresponding to the optimal value according to the previous obtained result), landscape disposition, and central inverter configuration. After a pitch of approximately $10 \mathrm{~m}$ has been attained, the grid injected energy is maximum and stays steady for higher pitch values.

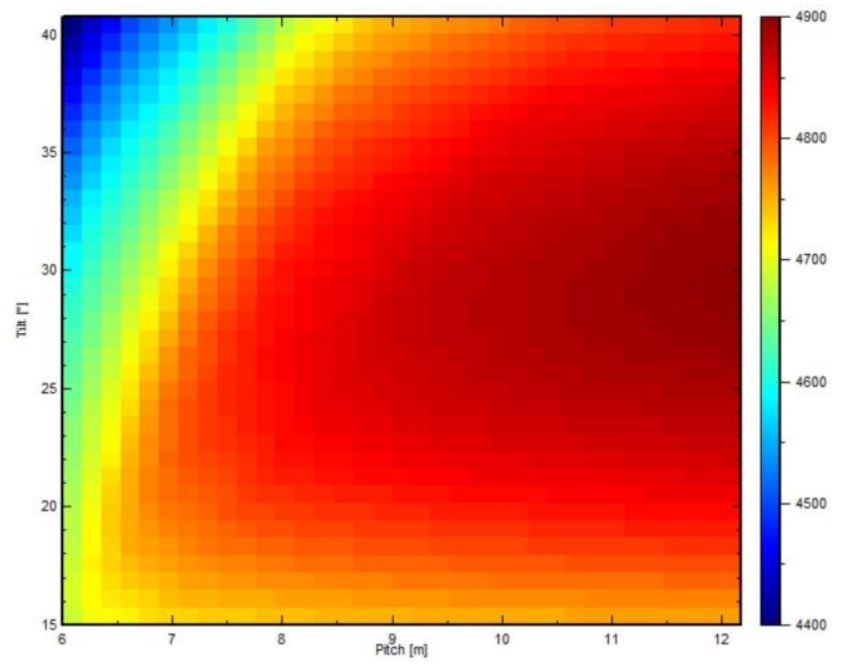

Figure 5. Sub-array grid injected energy (MWh) as a function of pitch and tilt; landscape; central inverter. 


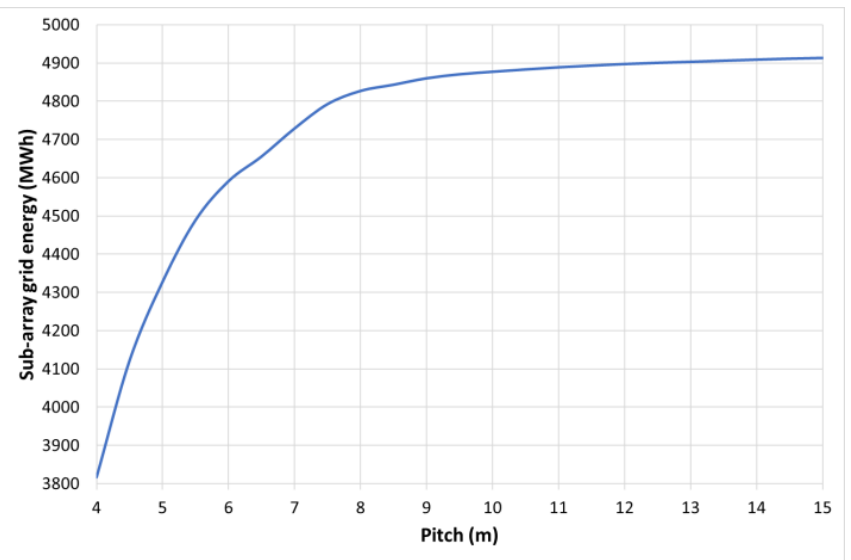

Figure 6. Sub-array grid injected energy as a function of pitch; $30^{\circ}$ tilt angle; landscape; central inverter.

Lastly, the azimuth angle was varied from $-15^{\circ}$ to $15^{\circ}$ to understand the direction the solar modules should face for the energy yield to be maximized. The maximum values of produced energy were obtained for a tilt of $30^{\circ}$, pitch equal to $9.65 \mathrm{~m}$, and for an azimuth angle of zero degrees (southern orientation), which confirms the common knowledge about this subject.

\subsection{Single-Axis Tracking System}

Simulations were carried out considering a single-axis tracking system with and without the employment of the backtracking strategy. Hence, the influence of both strategies was assessed with respect to the sub-array grid energy output and shading losses. Figure 7 shows the obtained results as a function of the pitch between rows for the landscape disposition and central inverter configuration. As one may notice, the backtracking strategy shows to be more advantageous in relation to the case in which it is not employed, leading to slightly greater values of grid energy yield output.

The shading losses per unit area associated with backtracking versus non-backtracking are included in the same graph, being the scale presented on the right side of the figure. A smaller value of pitch, together with the non-implementation of the backtracking strategy leads to greater shading losses' values, stemming mainly from the lack of incident beam component on the collector plane (visible shades). On the other hand, when the backtracking strategy is employed, there are no beam shading losses, since the collector plane constantly modifies the angle depending on the sun height. In this latter case, the shading losses arise mainly due to the diffuse and albedo component, which do not vary significantly along the pitch.

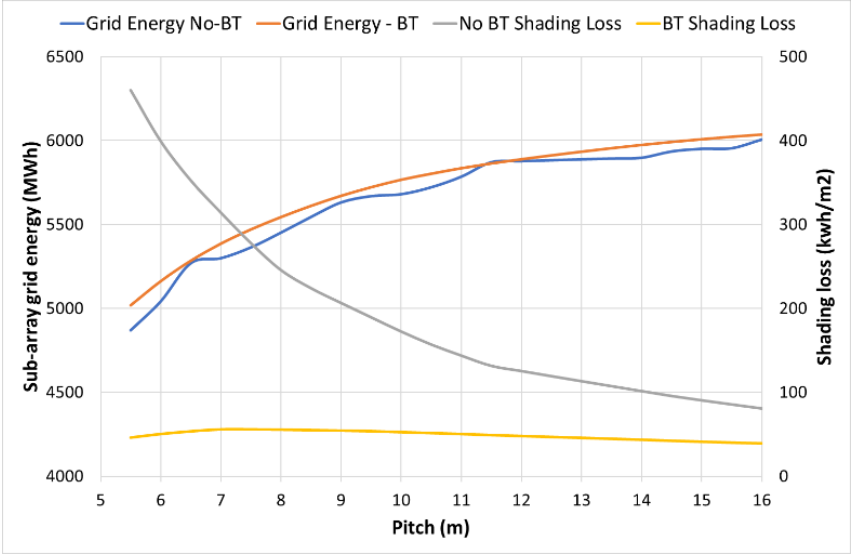

Figure 7. Sub-array grid injected energy and shading loss as a function of pitch; backtracking (BT) vs. no backtracking; landscape; central inverter. 
For the pitch between rows to be set, one may notice that for higher pitches than $9.65 \mathrm{~m}$, the sub-array grid-injected energy stays almost constant. So, it was decided to keep the pitch equal to the one corresponding to the fixed tilt system $(9.65 \mathrm{~m})$. As seen in Figure 7, for the SAT system, the pitch could be higher, but the $9.65 \mathrm{~m}$ pitch, while not compromising the grid-injected energy, enables occupying less terrain, for which other uses can be found.

\subsection{Systems' Performance Comparison}

Along the previous sections, both fixed tilt and SAT systems' performances were improved in the extent that relevant parameters were varied aiming at maximizing the energy yield. Additionally, both these systems were simulated with the already mentioned central and string inverters to conclude about their effect in the energy output.

Table 3 summarizes the grid-injected energy obtained results considering $9.65 \mathrm{~m}$ pitch for the different inverter configurations and landscape/portrait dispositions. The single-axis tracking system proves to clearly generate a greater amount of energy in comparison with the fixed tilt system. For instance, for the String- $105 \mathrm{~kW}$ configuration, landscape disposition, the SAT produces $16.7 \%$ more energy than the fixed tilt. In addition, using string inverters, for instance the String- $105 \mathrm{~kW}$, produces more $2.7 \%$ energy than the central inverter configuration for the SAT system in portrait disposition. This might be explained due to the beam shading, which is responsible for the strings' mismatch connected to the same MPPT (Maximum Power Point Tracker). As a string inverter possesses many more available MPPT inputs than a central inverter, the beam shading (and consequently the mismatch) is better circumvented. We finally note that portrait dispositions produce slightly more energy than landscape, in a SAT system, the opposite being observed for fixed tilt (as demonstrated above).

The overall configuration that leads to the maximum energy produced is using a SAT system of $9.65 \mathrm{~m}$ pitch with String- $105 \mathrm{~kW}$ inverters in portrait disposition.

\subsection{Economic Viability}

The PV park layout was undertaken (using AutoCAD) to dispose the different equipment over the given available area. Thus, for every situation, the different cables were sized, and their length was found, having been useful for a subsequent price estimate. Data related to the system components' costs (Tables A1 and A2), cables' price estimate (Table A3), and each system's O\&M costs (Table A4) are presented in Appendix A (June 2019 values). We note that the costs for the SAT system were considered to be $10 \%$ higher than for fixed tilt, as per indication of the PV market suppliers. A discount rate of $6 \%$ and 25-year lifetime were considered. In addition, a degradation factor of $0.4 \% /$ year was included when computing the grid-injected energy to account for the panels aging. Moreover, additional losses due to modules mismatch were considered. The levelized cost of energy (LCOE) for both studied systems were calculated while employing the three different types of inverters and both modules' dispositions. The results are shown in Table 4. We note that the computed LCOE does not include the grid connection costs, because in the case study under analysis, the point of common coupling was close to the PV park.

Table 3. Grid-injected energy; $9.65 \mathrm{~m}$ pitch; String-185 kW = SUN2000-185KTL; tring-105 kW = SUN2000-105KTL. SAT: single-axis tracking.

\begin{tabular}{ccccc}
\hline Tracking & Inverter Type & Tilt Angle & \multicolumn{2}{c}{ Grid Injected Energy (MWh) } \\
& & & Landscape & Portrait \\
\hline \multirow{3}{*}{ Fixed tilt } & Central & \multirow{2}{*}{$30^{\circ}$} & 38,996 & 38,952 \\
& String-185 kW & & 40,315 & 40,269 \\
& String-105 kW & & 40,364 & 40,318 \\
\hline \multirow{2}{*}{ SAT } & Central & & 45,812 & 45,898 \\
& String-185 kW & $-55^{\circ}$ to $55^{\circ}$ & 47,026 & 47,067 \\
& String-105 kW & & 47,090 & 47,131 \\
\hline
\end{tabular}


Table 4. Levelized cost of energy (LCOE) (€/MWh).

\begin{tabular}{cccc}
\hline Tracking & Inverter Type & Landscape & Portrait \\
\hline \multirow{3}{*}{ Fixed tilt } & Central & 35.06 & 35.07 \\
& String-185 kW & 34.55 & 34.57 \\
& String-105 kW & 34.56 & 34.58 \\
\hline \multirow{2}{*}{ SAT } & Central & 32.38 & 32.29 \\
& String-185 kW & 32.28 & 32.23 \\
& String-105 kW & 32.29 & 32.24 \\
\hline
\end{tabular}

As reflected in Table 4, the SAT system LCOE shows to be significantly lower relative to the fixed tilted regardless of the configuration type (portrait/landscape). Thus, even though the tracking system is more expensive than the fixed tilt one, the energy produced is significantly higher to offset the initial investment. Based on both current MIBEL (Iberian Electricity Market) average market price ( $50 € / \mathrm{MWh}$ ) and on the data from Table 4, the competitiveness of this type of technology is clear.

From the same table, one can perceive that in the fixed tilt system, the landscape configuration leads to slightly better results. So, in this case, the higher energy produced is sufficient to compensate the greater cables' investment. On the other hand, the portrait configuration has shown to be slightly cheaper in the case of the single-axis tracking system. The difference between these two systems might be justified due to the near shadings, which hinge on both solar panels' configuration and tracking system. In addition, for both tracking type of systems, the string inverters have proven to perform slightly better, leading to lower values of LCOE. More specifically, the String- $185 \mathrm{~kW}$ inverter, portrait disposition, has proved to achieve the lowest LCOE. This is because despite producing less energy (see Table 3), the String- $185 \mathrm{~kW}$ is cheaper (see Table A2).

Through the obtained results, one can conclude that the most suitable solution is the single-axis tracking system rather than the fixed tilt one. Furthermore, 112 SUN2000-185KTL string inverters should be employed in total, enabling the connection of 8 sub-arrays composed of 280 strings of 29 modules (Hanwha QCells 370W) each. Additionally, the system should use the backtracking strategy as well as the modules disposed in portrait.

\section{Conclusions}

The main goal of this paper was to investigate the maximization of the energy output of a $24 \mathrm{MWp}$ grid-connected solar PV park. PVSyst software was chosen as the decision support tool software. PVSyst was a crucial tool as it allowed to run successive simulations for each considered parameter, enabling individual assessment. Hence, through an automatic process of running the software a number of times in a row, one could perform a sensitivity analysis and assess the influence of each parameter on the energy yield maximization. Additionally, an economic analysis was carried out so that each scenario viability could be evaluated in a long-term period. Initially, all available PV modules were combined with each central inverter so that the most suitable combination module/inverter could be selected based on the maximum energy output criteria. Moreover, two different string inverters were also included in the study to assess their possible advantage relatively to the central inverter usual option.

After the modules and inverters have been chosen, two different types of system (fixed tilt and single-axis tracking) were considered, and significant parameters were varied to perceive how differently each one of them influences the energy output. Regarding the fixed tilt system, the module configuration (portrait/landscape) was analyzed, which allowed to conclude that the landscape configuration leads to slightly greater energy yields on account of near shadings. Concerning the single-axis tracking system, the backtracking strategy was assessed and concluded to be slightly advantageous. It was determined that for the String- $105 \mathrm{~kW}$ configuration and landscape disposition, the SAT produces $16.7 \%$ more energy than the fixed tilt. In addition, using string inverters, for instance 
the String- $105 \mathrm{~kW}$, produces more $2.7 \%$ energy than the central inverter configuration for the SAT system in portrait disposition.

The PV park layout was carried out in two different phases. At first, the devices' distribution and connections were undertaken based on the respective modules and inverters' data sheets, which was crucial to properly decide the required equipment quantity. Secondly, the different equipment was disposed over the available area (using AutoCAD), allowing the different cables' lengths to be estimated for each different case scenario. Finally, the cables' sizing was undertaken as well as their prices' estimates, having been prominent for a subsequent economic analysis. The LCOE was obtained for 12 different cases in which, for both fixed tilt and SAT, central and string inverters and portrait/landscape disposition were considered. The lowest LCOE achieved was $32.23 € / \mathrm{MWh}$, which is a clear indication of the competitiveness of the solar PV technology when we bear in mind that the current average MIBEL spot price is around $50 € /$ MWh.

Throughout this study, a method was developed to solve the optimization problem of a utility-scale solar PV park, contributing to its performance improvement as well as to the choice of the most suitable devices and parameters to employ. Based on the obtained results and among the wide range of possibilities through which this study has come across, one may conclude that the most viable solution (lower LCOE) is the one employing a single-axis tracking system together with a backtracking strategy as well as with the modules disposed in portrait configuration. A comparison between the considered central/string inverters together with an economic analysis has shown that the string inverter is the best alternative to employ as it does not just provide a better cost per unit of energy as well as it allows to be easily replaced whenever it is needed.

One important conclusion of our work is the best performance of the novel string inverters as compared to the traditional central inverter configuration. This is a result that confirms the new trend in using string inverters in big solar PV parks, which is currently far from being a current practice.

Author Contributions: Conceptualization, M.S., R.C. and M.B.; methodology, M.S., R.C. and M.B.; software, M.S.; validation, M.S.; formal analysis, M.S., R.C. and M.B.; investigation, M.S.; resources, M.S. and M.B.; data curation, M.S.; writing — original draft preparation, M.S.; writing—review and editing, R.C.; visualization, M.S. and M.B.; supervision, R.C. and M.B.; project administration, M.B.; funding acquisition, R.C. All authors have read and agreed to the published version of the manuscript.

Funding: This work was supported by national funds through Fundação para a Ciência e Tecnologia (FCT) under project UIDB/50021/2020.

Acknowledgments: The authors deeply acknowledge Sotécnica (Vinci Energies) for providing crucial data enabling this study to be successfully completed.

Conflicts of Interest: The authors declare no conflict of interest.

\section{Appendix A}

Table A1. Fixed tilt components' cost (central inverter configuration and portrait disposition).

\begin{tabular}{ccc}
\hline Item & $\mathbf{\%}$ & $\mathbf{E / W p}$ \\
\hline PV Modules & 51 & 0.306 \\
Support Structures & 7 & 0.042 \\
Accessories & 16 & 0.096 \\
Central Inverters & 9 & 0.054 \\
Elect. and Mech. Installation & 15 & 0.090 \\
Project and Commissioning & 1 & 0.006 \\
Control and monitoring system & 1 & 0.006 \\
\hline
\end{tabular}

Table A2. String inverters costs.

\begin{tabular}{cc}
\hline Inverter & $\mathbf{E / W p}$ \\
\hline String-105 kW & 0.0706 \\
String-185 kW & 0.0674 \\
\hline
\end{tabular}


Table A3. Cables' total cost.

\begin{tabular}{ccc}
\hline Inverter & Disposition & Cost $(\boldsymbol{(})$ \\
\hline Central & Landscape & 134,600 \\
& Portrait & 122,504 \\
String-105kW & Landscape & 262,790 \\
& Portrait & 250,694 \\
String-185kW & Landscape & 307,251 \\
& Portrait & 295,155 \\
\hline
\end{tabular}

Table A4. Fixed tilt and Single-axis tracking operation and maintenance costs.

\begin{tabular}{ccc}
\hline System & Inverter & $\boldsymbol{\epsilon / W p}$ (Annual) \\
\hline Fixed tilt & Central & 0.007 \\
& String & 0.00652 \\
Single axis & Central & 0.00714 \\
& String & 0.00665 \\
\hline
\end{tabular}

\section{References}

1. Kandasamy, C.; Prabu, P.; Niruba, K. Solar potential assessment using PVsyst software. In Proceedings of the 2013 International Conference on Green Computing, Communication and Conservation of Energy (ICGCE), Chennai, India, 12-14 December 2013; pp. 667-672.

2. Raj, A.; Gupta, M.; Panda, S. Design simulation and performance assessment of yield and loss forecasting for $100 \mathrm{kWp}$ grid connected solar PV system. In Proceedings of the 2nd International Conference on Next Generation Computing Technologies, Dehradun, Uttrakhand, India, 14-16 October 2016.

3. Yadav, P.; Kumar, N.; Chandel, S.S. Simulation and performance analysis of a $1 \mathrm{kWp}$ photovoltaic system using PVsyst, 2015. In Proceedings of the International Conference on Computation of Power, Energy, Information and Communication, Chennai, India, 22-23 April 2013.

4. Kumar, B.S.; Sudhakar, K. Performance evaluation of $10 \mathrm{MW}$ grid connected solar photovoltaic power plant in India. Energy Rep. 2015, 1, 184-192. [CrossRef]

5. Jamil, I.; Zhao, J.; Zhang, L.; Jamil, R.; Rafique, S.F. Evaluation of energy production and energy yield assessment based on feasibility, design, and execution of 3x50MW grid-connected solar PV pilot project in Nooriabad. Int. J. Photoenergy 2017, 2017, 1-18. [CrossRef]

6. Verma, A.; Singhal, S. Solar PV performance parameter and recommendation for optimization of performance in large scale grid connected solar PV plant-case study. J. Energy Power Sources 2015, 2, 40-53.

7. Mermoud, A. Optimization of row-arrangement in PV systems, shading loss evaluations according to module positioning and connections. In Proceedings of the 27th European Photovoltaic Solar Energy Conference, Frankfurt, Germany, 24-28 September 2012.

8. Mermoud, A. A tool to optimize the lay-out of ground-based PV installations taking into account the economic boundary conditions. In Proceedings of the 29th European Photovoltaic Solar Energy Conference, Amsterdam, The Netherlands, 22-26 September 2014.

9. Muhammad, Z.; Rosselan, B.; Sulaiman, S.I.; Musirin, I. Sizing optimization of large-scale grid-connected photovoltaic system using cuckoo search. Indones. J. Electr. Eng. Comput. Sci. 2017, 8, 169-176.

10. Kerekes, T.; Koutroulis, E.; Sera, D.; Teodorescu, R.; Katsanevakis, M. An optimization method for designing large PV plants. IEEE J. Photovolt. 2013, 3, 814-822. [CrossRef]

11. Rhodes, J.D.; Upshaw, C.R.; Cole, W.J.; Holcomb, C.L.; Webber, M.E. A multi-objective assessment of the effect of solar PV array orientation and tilt on energy production and system economics. Sol. Energy 2014, 108, 28-40. [CrossRef]

12. Hassan, Z.A.; Garni, A.A.; David, W. Optimal orientation angles for maximizing energy yield for solar PV in Saudi Arabia. Renew. Energy 2019, 133, 538-550.

13. Afanasyeva, S.; Bogdanov, D.; Breyer, C. Relevance of PV with single axis tracking for energy scenarios. Sol. Energy 2018, 173, 173-191. [CrossRef] 
14. Braun, H.; Buddha, S.T.; Krishnan, V.; Tepedelenlioglu, C.; Spanias, A.; Banavar, M.; Srinivasan, D. Topology reconfiguration for optimization of photovoltaic array output. Sustain. Energy Grids Netw. 2016, 6, 58-69. [CrossRef]

15. He, F.; Zhao, Z.; Yuan, L. Impact of inverter configuration on energy cost of grid-connected photovoltaic systems. Renew. Energy 2012, 41, 328-335. [CrossRef]

16. Sakthivel, R.; Muralitharan, K.; Shi, Y. Multiobjective optimization technique for demand side management with load balancing approach in smart grid. Neurocomputing 2016, 177, 110-119.

17. Li, H.; Guo, S.; Li, C.; Sun, J. A hybrid annual power load forecasting model based on generalized regression neural network with fruit fly optimization algorithm. Knowl.-Based Syst. 2013, 37, 378-387. [CrossRef]

18. Bessa, R.J.; Trindade, A.; Miranda, V. Spatial-Temporal Solar Power Forecasting for Smart Grids. IEEE Trans. Ind. Inform. 2015, 11, 232-241. [CrossRef]

19. Corizzo, R.; Pio, G.; Ceci, M.; Malerba, D. DENCAST: Distributed density-based clustering for multi-target regression. J. Big Data 2019, 6, 43. [CrossRef]

20. Ceci, M.; Corizzo, R.; Malerba, D.; Rashkovska, A. Spatial autocorrelation and entropy for renewable energy forecasting. Data Min. Knowl. Discov. 2019, 33, 698-729. [CrossRef]

21. Abdel-Nasser, M.; Mahmoud, K. Accurate photovoltaic power forecasting models using deep LSTM-RNN. Neural Comput. Appl. 2019, 31, 2727-2740. [CrossRef]

22. Teodorescu, R.; Liserre, M.; Rodríguez, P. Grid Converters for Photovoltaic and Wind Power Systems; John Wiley Sons, Ltd.: Hoboken, NJ, USA, 2011.

23. Mondol, J.D.; Norton, B. Optimal sizing of array and inverter for grid-connected photovoltaic systems. Sol. Energy 2016, 80, 1517-1539. [CrossRef]

(C) 2020 by the authors. Licensee MDPI, Basel, Switzerland. This article is an open access article distributed under the terms and conditions of the Creative Commons Attribution (CC BY) license (http://creativecommons.org/licenses/by/4.0/). 